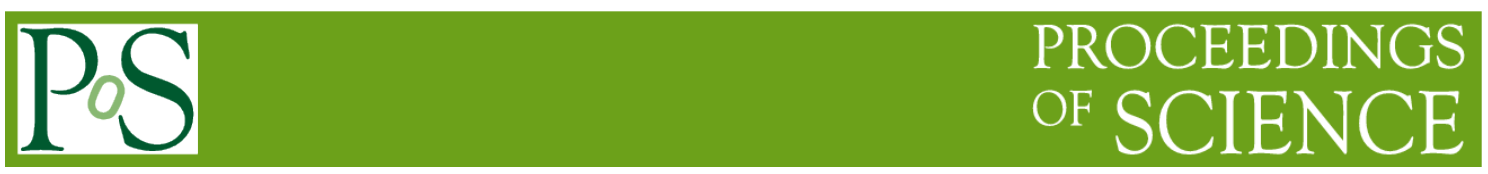

\title{
A Model for High and Low Frequency Oscillations in Black Hole Accretion Disks including Sgr A*
}

\section{Bernd Aschenbach ${ }^{1}$}

PR Vaterstetten

Mozartstrasse 8, 85591 Vaterstetten, Germany

E-mail: bernd.aschenbachet-online.de

\begin{abstract}
The frequency-mass relations of QPO black holes are explained by an epicyclic double resonance of $\Omega_{\varphi} / \Omega_{\theta}=3: 1$ and $\Omega_{\varphi} / \Omega_{r}=13: 1$ for a spin $a_{c}=0.99622$. Among the sources considered are the 3:2 frequency ratio, 'twin-peak' black holes and Sgr A*. The QPOs are suggested of generating resonant waves in the space bounded by the ISCO and the outer ergosphere surfaces. The physics are based on the non-Keplerian gravitational potential previously discovered in the Kerr metric. This characteristic is fundamental for the activities of most rapidly spinning black holes leading eventually to a resonance disaster of the black-hole/disk system.
\end{abstract}

Frontier Research in Astrophysics - III (FRAPWS2018)

28 May - 2 June 2018

Mondello (Palermo), Italy

${ }^{1}$ Speaker 


\section{Introduction}

Quasi-periodic oscillations (QPOs) have been observed in the light curves of black holes as temporal variations of the X-ray flux, in the near infrared and also in the optical bands. The measurements show a tight correlation between frequency $f$ and black hole mass $\mathrm{M}_{\mathrm{BH}}$ with $\left[f \cdot \mathrm{M}_{\mathrm{BH}}\right]=$ const. QPO objects of some solar masses, as well as some with thousands of solar masses up to supermassive black holes including Sgr A*, the black hole in the center of our galaxy, have been found.

QPOs are characterized by the appearance of distinct frequencies in the range of a few tens of $\mathrm{Hz}$ in the X-ray light curves of low-mass X-ray binaries (LMXB), with GX 5-1 being the first source of that kind discovered by van der Klis et al. (1985). Studying QPOs is promising information of the dynamics of the binary, the neutron star member in terms of its spin and mass, and the accretion disk around the neutron star. A summary of the low frequency QPO results obtained in the 1980's and 1990's can be found in the paper of van der Klis et al. (1995). In 1996 the first kHz QPO from the LMXB source 4U 1728-34 was reported by Strohmayer et al. A recent summary of $\mathrm{kHz}$ observations can be found in the paper of van Doesburgh et al. (2018).

Concerning the origin of the oscillations various models have been suggested, which, although differing in the physics involved, have in common, that the oscillations should arise close to the compact object in the binary, either a neutron star or a black hole candidate. Psaltis et. al (1999a) discovered a correlation between the low QPO frequency of a few tens of $\mathrm{Hz}$ and the lower frequency component of a QPO kHz pair. The correlation, which shows a frequency ratio of $\sim 10$, covers a range of about three orders of magnitude in frequency. Stella \& Vietri (1998, 1999) and Stella et al. (1999) suggested that strong gravity would significantly affect precession of the disk. Their Relativistic Precession Model (RPM), which uses periastron precession and nodal precession of a series of orbiting blobs on an inclined and eccentric disk, explains the observed frequencies and their correlation. The precession calculations are based on the use of the fundamental spherical coordinate frequencies; the radial frequency is also known as epicyclic frequency. They point out that their model would work both for a neutron star and a black hole candidate as the spin frequency is not essential for creating the observed frequencies and the correlation, in particular. This is contrasting the beat frequency model (BFM), for which the beat is between the orbital Kepler frequency and the spin frequency of the neutron star, which requires a coupling via a magnetosphere. A compilation of the various models and references as well as a in depth comparison of the BFM with the RPM is provided by Psaltis et al. (1999b).

The first pair of $\mathrm{kHz}$ QPOs with two frequencies coming in a 3:2 ratio was reported for the microquasar GRO J1655-40 by Strohmayer (2001). Abramowicz \& W. Kluźniak (2001) interpreted the appearance of the 3:2 frequency ratio as a parametric, non-linear resonance between the Kepler frequency and the radial epicyclic frequency and they calculated a spin value for the black hole candidate. Rezzolla et al. (2003) have suggested a physical model for 
the 3:2 twin peak resonance, which occurs because of seismic p-type oscillations of a fluid in a torus-like section of a non-Keplerian disk. They remark that the non-Keplerian fluid motion '... is required only very close to the black hole (the inner edge of the torus can in principle be located at the marginally bound orbit)' . This model would imply a fairly high spin, according to the authors. Lee et al. (2004) introduced an additional model which considers forced oscillations in the disk.

Pairs of 3:2 ratio $\mathrm{kHz}$ frequencies, the 'twin peak' oscillations were discovered subsequently for another three microquasars. Three of the four microquasar black holes are member of a binary system such that their masses are known from optical measurements. The mass of each of the black hole candidates is clearly above the most generous mass limit for a neutron star. Thereafter, the proponents of the epicyclic resonance theory studied every possible combination of any two of the three fundamental coordinate (epicyclic) resonances and calculated values for the spin of the involved microquasars using the measured mass (Abramowicz et al. 2004a, b). For additional references see the paper by Aschenbach (2004). Abramowicz et al. (2004a) noted a correlation between the measured mass of the black hole and the observed QPO frequencies, which would suggest a strong gravity origin of these oscillations. Using the epicyclic resonance theory I found coupled epicyclic resonances with a frequency ratio of 3:2 and 3:1 for a single spin value of $a=0.99616$ originating at two different radii. The coupling is enforced by the requirement that the two radii would measure two commensurable Keplerian orbits. It turns out that for such a constraint there exists only one solution with a 3:1 resonance between the two Keplerian frequencies (Aschenbach, 2004).

Since then the number of black holes with periodicity in the light curve has grown but the total number of QPO objects of the 3:2 frequency class remains still fairly small in view of the overwhelming number of black hole candidates. These QPO sources continue to be special and appear to have some characteristics which are specific. Smith et al. (2018) have summarized the QPO observational data related to black holes with clear periodicities. A copy of their Figure 4 is shown in Fig. 1. Some sources follow a line representing the 3:2 $\left[f \cdot \mathrm{M}_{\mathrm{BH}}\right]$-relation identifying the so-called HFQPOs (HF). There is no doubt that the 3:2 resonance can be attributed to strong gravity and black holes. The excitation mechanism of the 3:2 QPOs is unknown and whether the constraint by commensurable orbits and an associated dedicated single spin of the black hole are essential has remained an open question.

A second $\left[f \cdot \mathrm{M}_{\mathrm{BH}}\right]$-relation has been established at almost two orders of magnitude lower frequencies, the LFQPO (LF). There are sources which show a single HFQPO or a single LFQPO period, some show the 3:2 frequency ratio at HF and there are sources with both HF and LF (Smith et al., 2018), and it is therefore and because of the much higher HF/LF frequency ratio that this HF/LF correlation is not identical with the Psaltis et al. (1999) HF/LF correlation observed in neutron stars. Smith et al. (2018) note that there is no explanation for the LFQPOs. Below I present a model by which the LFQPOs can be explained by epicyclic resonances as well. 


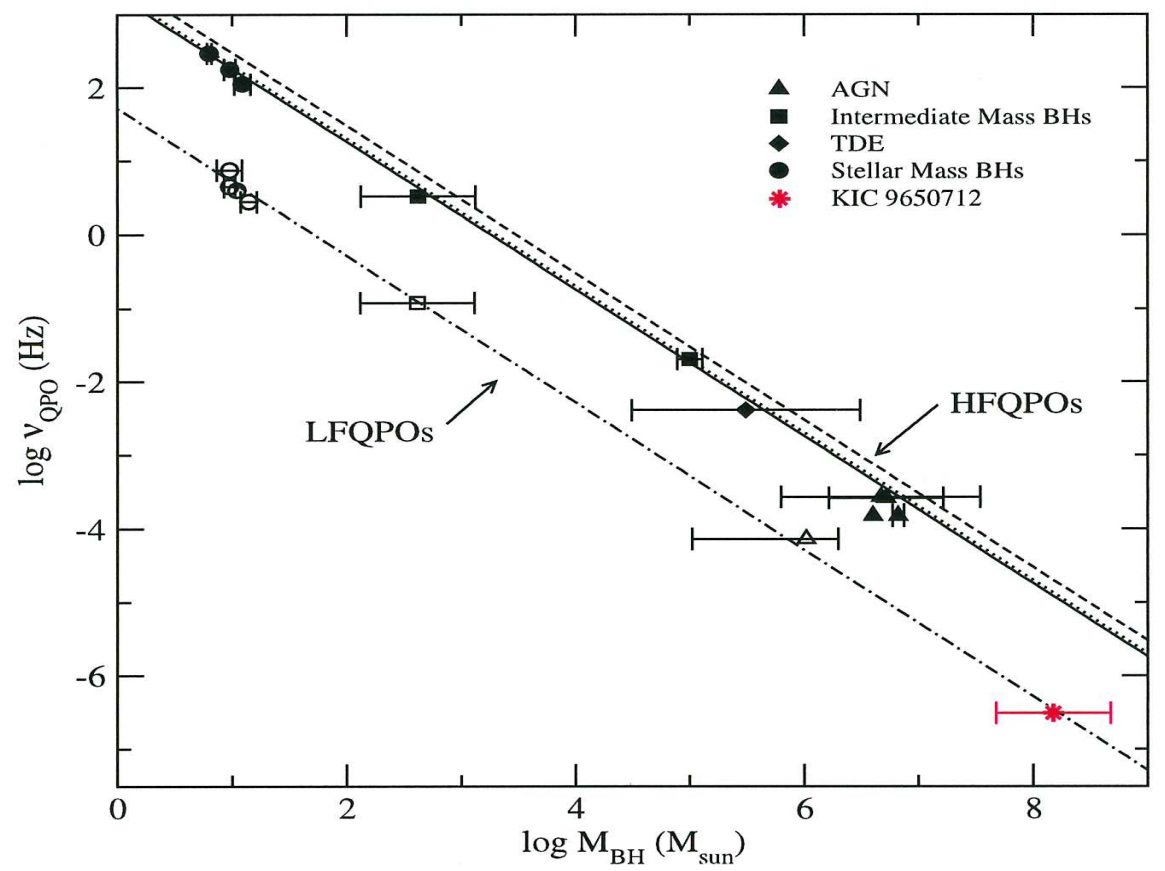

Figure 1: Black hole QPO frequency-mass relation (taken from Smith et al., 2018). The solid line shows the best-fit to the HFQPO related observations by Remillard \& McClintock (2006). The dashed line is the prediction of an epicyclic oscillation model for a 3:1 resonance (Eq. 4) and the dotted line for a 3:2 resonance (Eq. 5) by Aschenbach (2004). The dot-dashed line represents the linear regressive fit to the LFQPO data by Smith et al. (2018). The HFQPOs data and lines show the lower of the 3:2 twin-peak frequencies.

\section{Algebra of the epicyclic frequency relations}

There are three fundamental gravitationally induced oscillation modes in accretion disks around a massive and compact object, including black holes. These are the Kepler frequency $\Omega_{\varphi}$ and the perturbation eigenfrequencies along the polar direction $\Omega_{\theta}$ and the radial direction $\Omega r$. Rotation around the polar z-axis marks the orbital plane. A mass pushed slightly off the orbit by a small amount will oscillate up and down and back and forth with respect to its position on the circle. Therefore these oscillations are also called epicyclic. The Kepler frequency and the epicyclic frequencies depend on three parameters, which are the mass $\mathrm{M}$ of the central object, a black hole in this context, its spin $a$, and the radius $r$ of the orbit.

Equations 1 to 3 show the relations describing the frequencies $\Omega$ in standard notation with $\mathrm{c}=\mathrm{G}=\mathrm{M}=1$.

$\Omega_{\varphi}=\left(r^{3 / 2}+a\right)^{-1}$

$\Omega \theta^{2}=\Omega \varphi^{2}\left(1-4 a / r^{3 / 2}+3 a^{2} / r^{2}\right)$

$\Omega_{r}^{2}=\Omega_{\varphi}^{2}\left(1-6 / r+8 a / r^{3 / 2}-3 a^{2} / r^{2}\right)$

with $\Omega_{\varphi} \geq \Omega_{\theta}>\Omega_{r}$. Unlike $\Omega_{\varphi}$ and $\Omega_{\theta}$, the radial epicyclic frequency $\Omega_{r}$ can take the value 
$\Omega_{r}=0$, which defines the radius $r$ isco of the innermost (marginally) stable circular orbit (ISCO). Physical length scales are in units of the gravitational radius $r_{g}=\mathrm{GM} / \mathrm{c}^{2}$ and angular frequencies $\Omega$ are in units of $\mathrm{c}^{3} / \mathrm{GM}$. The conversion of mass normalized angular frequency $\Omega$ to observed frequency $f$ is obtained through $f=\Omega \times \mathrm{c}^{3} /(2 \pi \mathrm{GM})$.

\section{Epicyclic resonances in the ergosphere}

In 2004 I have shown that for the same spin value $a=0.99616$ two twin-peak resonances with $\Omega_{\theta} / \Omega_{r}=3: 1$ and $\Omega_{\theta} / \Omega_{r}=3: 2$ exist, which means occurring of a total of four frequencies. The 3:1 resonance frequencies originate from an orbit with radius $r_{31}$ and the 3:2 resonance frequencies originate from an orbit with radius $r_{32}$. The two orbits are commensurable orbits in the classical sense that their Kepler frequencies are related through $\Omega_{\varphi}\left(r_{31}\right)=\mathrm{n} \cdot \Omega_{\varphi}\left(r_{32}\right)$ with $\mathrm{n}$ a natural number. From Eq. $1-3$ it is derived that there is only one solution with $\mathrm{n}=3$, i.e, $\Omega_{\varphi}\left(r_{31}\right) / \Omega_{\varphi}\left(r_{32}\right)=3: 1$. The data are $a=a b=0.99616, r_{31}=1.546$ and $r_{32}=3.919$ (Aschenbach, 2004). With $\Omega \theta>\Omega_{r}$ it follows that the upper frequency $f_{\text {up }}$ of the twin frequencies corresponds to $\Omega \theta$. Accordingly the following relations hold for $\mathrm{M}$ with $\mathrm{M}_{31}$ for the 3:1 and $\mathrm{M}_{32}$ for the 3:2 frequency ratio, respectively. The frequency $f$ is given in $\mathrm{Hz}$ throughout the paper.

$$
\begin{aligned}
& \mathrm{M}_{31} / \mathrm{M}_{\odot}=4603.3 / f_{\text {up }} \\
& \mathrm{M}_{32} / \mathrm{M}_{\odot}=3046.2 / f_{\text {up }}
\end{aligned}
$$

In 2004, three twin-peak QPO objects, low mass X-ray binaries, were known with their black hole masses determined by their motion as member of a binary system: GRO J1655-40 ( $f$ $=450 / 300 \mathrm{~Hz})$, XTE J1550-564 $(f=276 / 184 \mathrm{~Hz})$ and GRS 1915+105 $(f=168 / 113 \mathrm{~Hz})$. I have calculated the masses from the measured frequencies using Eq. 5, and in all three cases the mass agrees with the measured value within the quoted measurement error range. In 2006, Remillard and McClintock used the binary measured mass and the measured frequencies of these three objects and published an empirical best fit to the data which is $\mathrm{M}=951 / f$. $f$ is supposed to be a so-called fundamental frequency which is related to $f_{\text {up }}$ by $f_{\text {up }}=3 \cdot f$, such that the Remillard \& McClintock best fit is equivalent to $\mathrm{M} / \mathrm{M}_{\odot}=2853 / f_{\text {up }}$. The best-fit constant in this relation differs from that predicted by the commensurability hypothesis (Eq. 5) by $<7 \%$.

As pointed out before orbit commensurability leads to selection of just one spin. A confirmation of the commensurability hypothesis hinges on the detection of the 3:1 resonance. But after more than 15 years of observations a 3:1 QPO has apparently not been found, which seems to have made orbital commensurability less likely to be a fundamental constraint for the occurrence of 3:2 QPOs, although the frequency ratio of 3:1 for $\Omega \theta / \Omega_{r}$ exists in the Kerr metric, 
and the 3:2 QPO seems to work fine. Apparently, the 3:1 resonance does not lead to a modulation of the photon flux. However, the resonance is generated in the ergosphere and processes in the ergosphere are known to be capable of extracting energy from the black hole like the Penrose mechanism, such that energy can leave the ergosphere. A fine-mesh search in the ergosphere for further resonances between the coordinate (epicyclic) frequencies has disclosed another multiple resonance pattern, which appears at $a_{c}=0.99622$ with $\Omega_{\varphi} / \Omega_{\theta}=3: 1$, $\Omega_{\varphi} / \Omega_{r}=13: 1$ at $r_{133}=1.39221$.

The resonance $\Omega \theta / \Omega_{r}=13: 3$ provides the $\left[f \cdot \mathrm{M}_{\mathrm{BH}}\right]$-relation (6) with the observed frequency $f_{\text {up }}$ attributed to $\Omega \theta . \Omega$ which is the highest frequency among the three frequencies is likely not to be observed (c.f. Section 6).

$\mathrm{M}_{133} / \mathrm{M}_{\odot}=4074.9 / f_{\text {up }}$

Unlike the frequencies $\Omega_{\theta}$ and $\Omega_{r}$ for $a=a_{b}$, the frequencies $\Omega_{\theta}$ and $\Omega_{r}$ for $a=a_{c}$ have probably been observed as QPOs. A candidate source is Sgr A*. Further evidence for an additional $a=a_{c}$ based epicyclic resonance is provided by the LFQPO mass-frequency relation shown in Fig. 1, which can be explained by a resonance between two commensurable radial epicyclic frequencies at $r_{0}$ and $r_{133}, r_{0}$ is identied in section 5.2.

There are two double or even multiple epicyclic resonances at two distinct, only slightly different spin states $a_{c}$ and $a_{b}$ in the ergosphere. Between these two spin states there appears something like a closed loop or cycle of resonances, i.e.: $\Omega_{\varphi} / \mathbf{\Omega}_{\theta}=3: 1\left(a=a_{c}\right), \Omega_{\theta} / \mathbf{\Omega}_{r}=3: 1$ ( $a=a b), \Omega_{r} / \Omega_{\varphi}=1: 13\left(a=a_{c}\right)$, which suggests some feedback maintaining a rate accuracy between the black hole spin states $\left(a_{c} \rightarrow a_{b} \rightarrow a_{c}\right)$ like in a clock. The ratio $a_{b} / a_{c}=1-1 / 15000$.

\section{Aschenbach effect and black hole spin oscillations}

In 2004 I have published the discovery of an anomalous orbital velocity behavior of the Kerr solution in its Boyer-Lindquist form (Aschenbach, 2004). Stuchlík et al., in their 2005 publication, have confirmed the effect and have called this behavior the Aschenbach effect. The unexpected effect relates to the orbital velocity $v_{\varphi}(r, a)$ and its radial gradient $\partial v_{\varphi}(r, a) / \partial r$. It has generally been believed that like in Newtonian mechanics also in the Kerr metric $\partial v \varphi(r, a) / \partial r<0$ for any $r$ and $a$. But for $a>0.9953 v_{\varphi}(r, a)$ shows a minimum/maximum structure for $r_{\text {min }}(a) \leq r \leq r_{\text {max }}(a)$ with $\partial v \varphi(r, a) / \partial r=0$ at $r=r_{\text {min }}(a)$ and $r=r_{\text {max }}(a)$, respectively, and $\partial v \varphi(r, a) / \partial r>0$ in between (Fig. 2.). At $r=r b(a), \partial v \varphi(r, a) / \partial r$ reaches a maximum value, and $\partial^{2} v_{\varphi}(r, a) / \partial r^{2}=0$. 


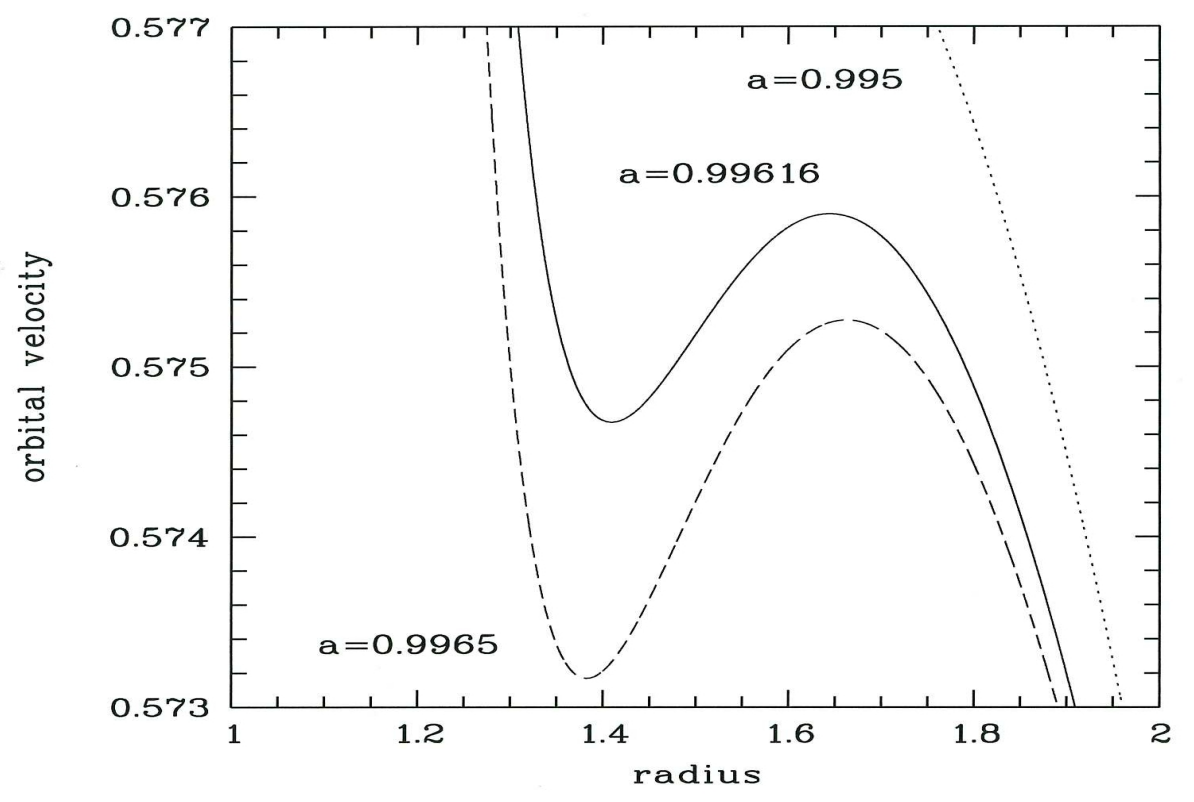

Figure 2: Anomaly of the radial dependence of the orbital velocity $v_{\varphi}$ for black hole spins with $a>0.9953$ (Aschenbach, $2004 \& 2007$ ). Radius is in units of $r_{g}$ and orbital velocity is in units of $\mathrm{c}$.

The effect appears in a region of the ergosphere with stable circular orbits, $r_{\text {min }}>r_{\text {isco, }}$, for $a>0.9953$. Still today no physical explanation of this effect has been published.

The fairly low value of $v_{\varphi}$ indicates a comparatively low value for the kinetic energy and a comparatively low value of the orbital angular momentum $l_{z}$ of the orbiting mass. The fraction of energy $\mathrm{E}$ and and angular momentum attributed to $v_{\varphi}$ is decreasing further with the orbiting mass approaching the ISCO (c.f. Fig. 2). Something appears to slow down the descent of the mass towards the black hole. I suggest that this occurs because of the presence of some torque with the torque axis located in the orbital plane, which is normal to the z-axis, the spinaxis of the black hole. The turning around the z-axis against the turning direction of the in-orbit torque axis, which make a $90^{\circ}$ angle with each other, leads to stress with stress-energy building up during descent of the accreting mass. The spin $a$ increases as well, the black hole is spinningup.

When the descending mass arrives at the ISCO surface the orbital angular momentum $\mathrm{l}_{\mathrm{z}}$ and the energy $E$ of the descending mass are $\mathrm{l}_{\mathrm{z}}=0$ and $\mathrm{E}=0$. Slightly below the ISCO $\mathrm{l}_{\mathrm{z}}<0$ and orbital angular momentum is being drawn from the black hole. The black hole is spinning down. The angular momentum released from the black hole will accelerate some fraction of the mass back to a higher orbit, i.e., $\partial v_{\varphi}(r, a) / \partial r>0$ and $\partial^{2} v_{\varphi}(r, a) / \partial r^{2}>0$, possibly up to $r=r b(a)$, where $\partial v_{\varphi}(\mathrm{r}, \mathrm{a}) / \partial \mathrm{r}$ reaches its maximum, or even higher up to $r=r_{\max }(a)$, where still $\partial v_{\varphi}(r, a) / \partial r>0$ but $\partial^{2} v_{\varphi}(r, a) / \partial r^{2}<0$. The location $r=r \max (a)$ with $\partial v_{\varphi}(r, a) / \partial r=0$ marks a point of unstable equilibrium, which would mean that as long as the reverse angular momentum does not take the split fraction of the mass beyond $r=r_{\max }(a)$ the cycle starts again with the descent bringing down more accreting mass. The rising of cells of mass/energy suggests an 
interpretation of convective motion as mass density and velocity are involved. This process would work as long as a $>0.9953$. It looks as if the space between the ISCO surface and $r=r_{\max }(a=0.9953)$, which is still within the outer ergosphere surface is being pressurized and heated. Some fraction of the accreting energy is used for doing the work. If eventually $r>r_{\max }(\mathrm{a}=0.9953)$ is reached the system black hole/accretion disk will break up and fractions or the total mass of the disk will be released in a fireball-burst due to centrifugal forces in a gravitational potential with $\partial v \varphi(r) / \partial r<0$.

I suggest that the purpose of the anomalous orbital velocity effect is to convert orbital kinetic energy in the space bounded by the ISCO and the outer ergosphere surfaces into pressure and temperature raising. The key feature of this model is the "split fraction of mass". If the stress-energy created by the two orthogonal rotations is somehow related to the gravitational stress-energy tensor, relativistic mass and pressure are expected to be created close to or on the ISCO surface.

\section{Comparison with observations}

\subsection{Sgr A* - the Galactic Centre Black Hole - revisited}

X-ray observations have revealed that Sgr A* shows flaring activity with occasionally very bright flares (Baganoff et al., 2001, Porquet et al., 2003). In the near-infrared bright flares were also discovered and a mean period of $16.8 \pm 2 \mathrm{~min}(1008 \pm 120 \mathrm{~s})$ has been reported (Genzel et al., 2003). Similar periods of $1173 \mathrm{~s}$ and $1117 \mathrm{~s}$ were suggested to have been found in X-ray light curves of both the quiescent and flaring state (Aschenbach et al., 2004). Some evidence for the presence of additional periods can be found in the data; but none of the claimed periods has been accepted as statistically convincing. A six day long, uninterrupted XMM observation (Porquet et al., 2008) of Sgr A* has shown a series of flares, which I have searched for a regular, periodic flare start-time. The same periods as in the earlier light curve period search were found, in particular a signal at $1104 \mathrm{~s}$ (Aschenbach, 2009).

Recently, Leibowitz (2018) has reported a 149 minute periodicity underlying the X-ray flaring process of Sgr A*, which he has called a 'pace maker'. This periodicity was found in XMM and Chandra data which covered the observations of the years $2000-2014$. More precisely, the periodicity is 0.103203 days or $8917 \mathrm{~s}$. The author suggests that this is the period of a low mass star or planetary object orbiting the black hole at a radius of about 6.6 Schwarzschild radii. Instead, I suggest that this period of $8917 \mathrm{~s}$ is twice the radial epicyclic period $\mathrm{P}_{r}$ to be associated with the double-resonance $\left[\Omega_{\varphi} / \Omega_{r}=13: 1, \Omega_{\varphi} / \Omega_{\theta}=3: 1, \Omega_{\theta} / \Omega_{r}=13: 3\right]$ for $a=a_{c}$ at $r=r_{133}$ described in the previous chapter, i.e., $\mathrm{P}_{r}=4458 \mathrm{~s}$, and the observed period of $\sim(1000-1100) \mathrm{s}$ would correspond to $\mathrm{P} \theta$. The observational data best matching results are $\mathrm{P}_{\varphi}=343 \mathrm{~s}\left(\mathrm{P}_{r} / \mathrm{P}_{\varphi}=13: 1\right), \mathrm{P}_{\theta}=1029 \mathrm{~s}\left(\mathrm{P}_{\theta} / \mathrm{P}_{\varphi}=3: 1\right)$ and $\mathrm{P}_{r}=4458 \mathrm{~s}$. Applying Eq. 6, the mass of Sgr $\mathrm{A}^{*}$ is $(4.19 \pm 0.12) \cdot 10^{6} \mathrm{M}_{\odot}$ and the spin $a=0.99622$. The relative small error reflects the repeatedly observed period range between $\sim(1000-1200) \mathrm{s}$. 


\subsection{Low frequency Quasi-periodic oscillations (LFQPOs)}

There are numerous possibilities which could explain the factor of two between the Leibowitz result and $\mathrm{P}_{r}$ used here. Period doubling (Rebusco et al., 2012) could be one solution; the analysis by Leibowitz may not exclude a 'pacemaker' with the periodicity of $\mathrm{P} r$ or $\mathrm{P}_{r} / 2$ being a 'true' harmonic of the periodicity found by Leibowitz. The question arises, whether the Leibowitz period is a fundamental period or whether it is some harmonic of a true fundamental period $\Omega_{r}\left(r_{0}\right)$ of the radial epicyclic mode at a radius $r_{0}$. Figure 1 shows that black holes with LFQPOs also follow a $\left[f \cdot \mathrm{M}_{\mathrm{BH}}\right]$-relation which comprises black hole masses from a few solar masses up to possibly more than $10^{8}$ solar masses. The model of epicyclic resonances presented in this paper shows that for the spin $a=a_{c}=0.99622$ an orbit radius $r_{0}$ exists at which $\Omega_{r}\left(r_{133}\right) / \Omega_{r}\left(r_{0}\right)=18: 1$ and $\Omega \theta\left(r_{0}\right) / \Omega_{r}\left(r_{0}\right)=70: 1$. The radius $r_{0}=1.30462$.

For comparison $r_{\text {isco }}=1.30434$. The values of $a, r_{0}$ and $\Omega_{r}\left(r_{0}\right)$ as a radial epicyclic frequency lead to the $\left[f-\mathrm{M}_{\mathrm{BH}}\right]-$ relation

$$
f(\mathrm{~Hz})=52.3 / \mathrm{M}_{\mathrm{BH}}
$$

$$
\text { with } \mathrm{M}_{\mathrm{BH}} \text { in } \mathrm{M}_{\odot} \text {. }
$$

The best-fit to the data by Smith et al. (2018) is $f(\mathrm{~Hz})=51.9 / \mathrm{M}_{\mathrm{BH}}$, with $\mathrm{M}_{\mathrm{BH}}$ in $\mathrm{M}_{\odot}$.

The small difference between $r_{\text {isco }}=1.30434$ and $r_{0}=1.30462$ and the realization of a period associated with $\Omega_{r}$ at $r_{0}$ suggests that $r_{0}$ is the physically lowest stable orbit rather than $r_{\text {isco }}$ which is the mathematically possible lowest circular stable orbit, or in other words, the marginally possible stable orbit, as it has originally been defined. In that sense the light curve of a black hole with $a=a_{c}$ could be modulated in time according to Eq. 7. The period length/time does not depend on the physical conditions prevailing in the accretion disk but just by the mass and spin of the black hole. For instance, for Sgr A* this period is calculated to $\mathrm{P}_{0}=0.927254 \mathrm{~d}$.

\section{Summary}

The results are summarized in Table 1. Frequencies are given for a one-solar mass black hole, the spin is $a=a_{c}=0.99622$ for the upper section, and $a=a b=0.99616$ for the lower section of the table, respectively. As pointed out before (Aschenbach, 2004) there are additional resonances between different modes engaging different orbits. Table 1 shows one of such resonant behavior across $r=r_{133}$ and $r=r_{32}$ by $\Omega \theta\left(r_{133}\right) / \Omega_{r}\left(r_{32}\right)=2: 1$. The entries of Table 1 show that frequency ratios close to 1:2, 1:3, 1:4, 2:3 and 3:4 may appear in the measurements. Table 1 can be used to disentangle the association with the appropriate frequency mode by their frequency ratios.

A particular case of resonance appears to be the resonance between $\Omega \theta$ for $\left[a=a b, r=r_{31}\right]$ and $\Omega \theta$ for $\left[a=a_{c}, r=r_{133}\right]$; the values for $\Omega \theta$ differ by just $11 \%$, such that the frequency related to $\Omega \theta$ might be changing by about that amount over an observation. Such a drifting of the $1008 \mathrm{~s}$ 
period of Sgr A* has been reported by Genzel et al. (2003), for instance, and it is compatible with the longer $\mathrm{X}$-ray periods of $\sim 1100 \mathrm{~s}$.

The orbital frequency $\Omega \varphi$ is likely not to be observed in the light curves. As long as the matter around the orbit is rather smoothly distributed there is no time variation. Even if a major blob of mass is moving along the Kepler orbit the observer will not see the Kepler frequency of the blob's motion but the light curve will show a regular but significantly shorter period than the Kepler period because of the almost extreme spin and proximity to the black hole (Cunningham \& Bardeen, 1972, Aschenbach, 2009). Table 1 shows that a 3:2 resonance may not necessarily be attributed to $a=a b$ but it could as well be attributed to the spin state of $a=a_{c}$. At those large radii associated with the 3:2 resonance the QPO resonance analysis is fairly insensitive to the spin state whereas for radii within the ergosphere it is.

Table 1: The QPO frequency spectrum of a black hole accretion disk $f(\mathrm{~Hz}) \cdot \mathrm{M}_{\mathrm{BH}}\left(\mathrm{M}_{\odot}\right)=$ const.

\begin{tabular}{|c|c|c|c|}
\hline \multicolumn{4}{|c|}{$a=0.99622, r_{\text {isco }}=1.30434$} \\
\hline$\underline{\text { orbit radius }\left(r_{\mathrm{g}}\right)}$ & epicyclic mode(s) & multiplicity & $f \cdot \mathrm{M}\left(\mathrm{Hz} \cdot \mathrm{M}_{\Theta}\right)$ \\
\hline$r_{0}=1.30462$ & $\Omega r$ & single & $52.3\left[\Omega_{r}\right]$ \\
\hline$r_{133}=1.39221$ & $\mathbf{\Omega}_{\varphi} / \mathbf{\Omega}_{r}=13: 1$ & double & $4074.9[\Omega \theta]$ \\
\hline & $\Omega_{\varphi} / \Omega_{\theta}=3: 1$ & & $940.4\left[\Omega_{r}\right]$ \\
\hline & $\Omega_{\theta} / \Omega_{r}=13: 3$ & & \\
\hline$r_{32}=3.91734$ & $\Omega_{\theta} / \mathbf{\Omega}_{r}=3: 2$ & double & $3046.2[\Omega \theta]$ \\
\hline & & & $2030.8\left[\Omega_{r}\right]$ \\
\hline & $a=0.99616, r_{\text {is }}$ & $=1.306$ & \\
\hline$r_{31}=1.546$ & $\Omega_{\theta} / \mathbf{\Omega}_{r}=3: 1$ & double & $4603.3[\Omega \theta]$ \\
\hline & & & $1534.4\left[\Omega_{r}\right]$ \\
\hline$r_{32}=3.919$ & $\mathbf{\Omega}_{\theta} / \mathbf{\Omega}_{r}=3: 2$ & double & $3046.2[\Omega \theta]$ \\
\hline & & & $2030.8\left[\Omega_{r}\right]$ \\
\hline
\end{tabular}

\section{Some concluding remarks}

It has been shown that the observed mass/frequency relations can be explained by resonant behavior between epicyclic oscillations of mass/energy in the very inner part of the accretion disk $1.304<r<4$, and $a>0.9953$. The LFQPOs originate at $r_{0}$ which is so close to $r_{\text {isco }}$ that $r_{0}$ 
is likely to mark the inner, still stable edge of the accretion disk, from which light is observed modulated with a frequency which is identical to the radial epicyclic mode frequency. On the ISCO surface $\mathrm{E}(r)$ and $\mathrm{l}_{\mathrm{z}}(r)$, i.e., the energy and the z-component of the angular momentum of matter flowing downstream the disk, both become zero. Notably, the same result for the value of $r_{\text {isco }}$ is obtained for $\Omega_{r}=0$, which could be interpreted as indicating that no oscillations in radial direction are possible anymore, since $\Omega_{r}$ has no solution for $r<r_{\text {isco }}$. For the Zero Angular Momentum Observer (ZAMO) the ISCO looks like a wall oscillating in radial direction while the spin is changing. The model presented in Section 4 may apply. Downstream matter is piling up against this wall and drives a wave upstream. With increasing retro-spin and $\partial v(r) / \partial r>0$ also an outer boundary at $\left[r=r_{\max }, \partial v(r) / \partial r=0\right]$ starts growing for $a>0.9953$, such that an inner and outer boundary within the ergosphere exist.

With increasing mass and spin/orbit angular momentum the black hole is approaching the extreme Kerr limit of $a=1$, beyond which accreting angular momentum is physically no longer possible. Angular momentum and mass start to be stored in the disk when $\mathrm{a}<1$, thus forming a binary system. Growing of the disk might last until the capacity of the storage region has been reached. The storage limit is defined by the point of unstable equilibrium marked by $\partial v\left(r=r_{\text {out }}\right) / \partial r=0$. For $r<r_{\text {out }}$ storage is possible. Beyond this point upstream mass/energy loss can occur because $\partial v(r) / \partial r<0$ with no outer edge. Such energy outflows can happen sporadically or can eventually comprise the entire disk. The result is an explosive separation of fractions or the entire disk. For the disk to be expelled it is to be lifted beyond the last stable orbit of zero angular momentum, which is six times the gravitational radius of the black hole.

The separation length is $\Delta r \sim 6 r_{\mathrm{BH}}-1 r_{\mathrm{BH}}=5 r_{\mathrm{BH}}$ or the separation energy required is $\Delta \mathrm{E} \sim 1 / 5 \mathrm{M}_{\mathrm{BH}}$. As the accretion disk takes up this amount of energy as kinetic energy the total available energy of the binary is split in two parts, which come at a ratio of 5:6, or 1:1.2.

The separation of the accretion disk from its hosting black hole would mimic the collision and subsequent explosion of two black holes, which encounter each other. According to Thorne (1974) it takes about $10^{7}$ years for a black hole to reach its limiting spin when accreting matter at a rate of the Eddington limit. Therefore such events could happen frequently, in contrast to random collisional encounters in space. If photons would be emitted at all from the expelled matter they would form a polar jet and, from the equatorial region, a rapidly rotating jet, with both emission regions extremely collimated because of the spin extremal. The mechanism would not be restricted to a black hole but would work as well for an extremely fast spinning neutron star with a comparatively massive and rapidly spinning disk. Summarizing I suggest to consider the majority of the observed gravitational wave events to be possibly the explosive separation of a black hole (neutron star) from its accretion disk rather than a random collision of two black holes or neutron stars.

The disruption of a black hole and its associated disk is the ultimate catastrophic event caused by excessive resonance increase. Along the way to that breaking state the system is likely to show activity/variability because of the spin up and spin down. In this way jets, winds, outflows, mass ejections and radiation flares on various scales may be created. This happens each time upstream angular momentum flow is crossing the point of unstable equilibrium.

I suggest that $\mathrm{Sgr} \mathrm{A}^{*}$ is a source in such a state, as all the phenomena resulting from such activity have been observed in the vicinity of $\mathrm{Sgr} \mathrm{A}^{*}$, including a major radiation flare 350 years 
ago (e.g., Koyama, 2018). Possibly even the mass contained in the molecular clouds in the immediate vicinity of Sgr A* might have been delivered through the spin up / spin down mechanism. At present the accretion disk of Sgr $\mathrm{A}^{*}$ is lacking mass/energy and the output in terms of radiation both on the quiescent level as well as in flares is low; the spin, though, is extreme.

Last but not least, I note that the substantial change of the gravitational potential with the spin is an intrinsic part of the Kerr solution of Einstein's field equations. It is a fundamental, if not vital property of gravity and it is this property which actually prevents and excludes the existence of black holes in the sense of ever in mass growing gravitational objects, which tend to merge creating even more massive black holes. On the contrary, one could view this 'catastrophic' event as a 'fireball' creating mass.

One could have the idea that the ISCO surface enclosing a cavity is a realization of Planck's black body of some radius and temperature. On the one hand, with on-going accreting of mass/energy the temperature of the ISCO surface rises, while, on the other hand, the surface of the ISCO gets smaller with increasing spin by accreting angular momentum. Cooling of the surface gets progressively difficult. Furthermore, partially embedded in the ergosphere, the ISCO surface cannot cool effectively by radiation. The two polar caps of the ISCO surface which are outside of the outer ergosphere surface make up less than $10 \%$ of the total ISCO surface area. The final event is a thermal runaway, by which the storage region in the ergosphere turns into an expanding "fireball" of the Fermi, Landau, Hagedorn type and it will cool to a temperature regime at which elementary matter is going to "freeze-out".

\section{References}

[1] M.A. Abramowicz \& W. Kluźniak 2001, A precise determination of black hole spin in GRO J1655-40, A\&A 374, L19 - L20.

[2] M.A. Abramowicz, W. Kluźniak, Z. Stuchlík \& G. Török 2004, The orbital resonance model for twin peak kHz QPOs - Measuring the black hole spins in microquasars, astro-ph/0401464.

[3] M.A. Abramowicz, W. Kluźniak, Z. Stuchlík \& G. Török 2004, Twin peak QPOs frequencies in microquasars and $\mathrm{Sgr} A^{*}$, 2004, In Proceedings of RAGtime 4/5 Workshops on black holes and neutron stars, 14-16/13-15 October 2002/2003, Eds: S. Hledík and Z. Stuchlík, held in Opava, Czech Republic; published by Silesian University in Opava, 12/2004, ISBN 80-7248-242-4, pp. 123.

[4] B. Aschenbach, N. Grosso, D. Porquet \& P. Predehl 2004, X-ray flares reveal mass and angular momentum of the Galactic Center black hole, A\&A 417, 71 - 78.

[5] B. Aschenbach 2004, Measuring mass and angular momentum of black holes with high-frequency quasi-periodic oscillations, A\&A 425, 1075 - 1082.

[6] B. Aschenbach 2007, An Overlooked Effect of General Relativity Applied to the Galactic Center Black Hole Sgr A*, JPhCS 66, 2039.

[7] B. Aschenbach 2009, Evidence for GR rotational frame-dragging in the light from the Sgr A* supermassive black hole, 2009arXiv:0911.2431A.

[8] F.K.Baganoff, M.W. Bautz, W.N. Brandt, G. Chartas, E.D. Feigelson, G.P. Garmire, Y. Maeda, M. Morris, G.R. Ricker, L.K. Townsley \& F. Walter 2001, Rapid X-ray flaring from the direction of the supermassive black hole at the Galactic Centre, Nature 413, 45 - 48. 
[9] J.M. Bardeen 1970, Kerr Metric Black Holes, Nature 226, 64 - 65.

[10] C.T. Cunningham \& J.M. Bardeen 1972, The optical Appearance of a Star Orbiting an Extreme Kerr Black Hole, ApJ 173, 137 - 140.

[11] R. Genzel, R. Schödel, T. Ott, A. Eckart, T. Alexander, F. Lacombe, D. Rouan \& B. Aschenbach 2003, Near-infrared flares from accreting gas around the supermassive black hole at the Galactic Centre, Nature 425, 934 - 937.

[12] K. Koyama 2018, Diffuse X-ray sky in the Galactic center, PASJ 70, 1- 50.

[13] W.H. Lee, M.A. Abramowicz \& W. Kluźniak 2004, Resonance in Forced Oscillations of an Accretion Disk and Kilohertz Quasi-periodic Oscillations, ApJ 603, L93 - L96.

[14] E. Leibowitz 2018, A 149 min periodicity underlies the X-ray flaring of Sgr $A^{*}, M N R A S$ 547, 3380 3390.

[15] D. Porquet, P. Predehl, B. Aschenbach , N. Grosso, A. Goldwurm, P. Goldoni, R.S. Warwick \& A. Decourchelle 2003, XMM-Newton observation of the brightest flare detected so far from $\operatorname{Sgr} A^{*}$, A\&A 407, L17 - L20.

[16] D. Porquet, N. Grosso, P. Predehl, G. Hasinger, F. Yusef-Zadeh, B. Aschenbach, G. Trap, F. Melia, R.S. Warwick, A. Goldwurm, G. Bélanger, Y. Tanaka, R. Genzel, K. Dodds-Eden, M. Sakano \& P. Ferrando 2008, X-ray hiccups from Sagittarius $A$ * observed by XMM-Newton. The second brightest flare and three moderate flares caught in half a day, A\&A 488, 549 - 557.

[17] D. Psaltis, T. Belloni \& M. van der Klis 1999a, Correlations in Quasiperiodic Oscillations and Noise Frequencies among Neutron Star and Black Hole X-ray Binaries, ApJ 520, 262 - 270.

[18] D. Psaltis, R. Wijnands, J. Homan, P.G. Jonker, M. van der Klis, M.C. Coleman, F.K. Lamb, E. Kuulkers, J. van Paradijs \& W.H.G. Lewin 1999b, On the Magnetic Beat-Frequency and LenseThirring Interpretations of the Horizontal-Branch Oscillation in the Z sources, ApJ 520, $763-775$.

[19] P. Rebusco, P. Moskalik, W. Kluźniak \& M.A. Abramowicz 2012, Period Doubling and non-linear resonance in the black hole IGR J17091-3624?, A\&A 540, L4 - L6.

[20] R. A. Remillard \& J.E. McClintock 2006, X-Ray Properties of Black-Hole Binaries , ARA\&A 44, 49-92.

[21] L. Rezzolla, S'i. Yoshida, T.J. Maccarone \& O. Zanotti 2003, A new simple model for high frequency quasi-periodic oscillations in black hole candidates, MNRAS 344, L37 - L41.

[22] K.L. Smith, R.F. Mushotzky, P.T. Boyd \& V.R. Wagoner 2018, Evidence for an Optical Lowfrequency Quasi-periodic Oscillation in the Kepler Light Curve of an Active Galaxy, ApJ 860, L10 L15.

[23] L. Stella \& M. Vietri 1998, Lense-Thirring Precession and QPOs in Low Mass X-ray Binaries, ApJ 82, L59 - L62.

[24] L. Stella, M. Vietri \& M. Morsink 1999, kHz Quasiperiodic Oscillations in Low-Mass X-ray Binaries as Probes of General Relativity in the Strong-Field Regime, Phys. Rev. Lett. 82, 17 - 20.

[25] L. Stella \& M. Vietri 1999, Correlations in the QPO Frequencies of Low Mass X-ray Binaries and the Relativistic Precession Model, ApJ 524, L63 - L66.

[26] T.E. Strohmayer, W. Zhang, J.H. Swank, A. Smale, L.Titarchuk, C. Day \& U. Lee 1996, Millisecond X-ray Variability from an Accreting Neutron Star System, ApJ 469, L9 - L12. 
[27] T.E. Strohmayer 2001, Discovery of a $450 \mathrm{~Hz}$ Quasi-periodic Oscillation from the Microquasar GRO J1655-40 with the Rossi X-ray Timing Explorer, ApJ 552, L49 - L53.

[28] Z. Stuchlík, P. Slaný, G. Török \& M.A. Abramowicz 2005, Aschenbach effect: Unexpected topology changes in the motion of particles and fluids around rapidly rotating Kerr black holes, ApJ 552, L49 - L53.

[29] K.S. Thorne 1974, Disc-Accretion onto a Black Hole. II. Evolution of the Hole, ApJ 191, 507 - 510.

[30] M. van der Klis, F. Jansen, J. van Paradijs, W.H.G. Lewin, E.P.J. van den Heuvel, J.E. Trumper \& M. Szatjno 1985, Intensity-dependent quasi-periodic oscillations in the X-ray flux of GX5-1, Nature 316, 225-230.

[31] M. van der Klis 1995, Rapid aperiodic variability in X-ray binaries, in 'X-ray Binaries', eds. W.H.G. Lewin, J. van Paradijs \& E.P.J. van den Heuvel, Cambridge University Press, p. 252-307.

[32] M. van Doesburgh, M. van der Klis \& S.M. Morsink 2018, The highest kHz QPOs in neutron star low-mass X-ray binaries, MNRAS 479, 426 - 435. 